\title{
A governance framework with permissioned blockchain for the transparency in e-tendering process
}

\author{
Mohammed Khaled Mustafa ${ }^{1^{*}}$ and Sajjad Waheed ${ }^{2}$ \\ Leading Telecommunication Operator in Bangladesh ${ }^{1}$ \\ Professor, Department of Information and Communication Technology, Mawlana Bhashani Science and Technology \\ University, Tangail, Bangladesh ${ }^{2}$
}

Received: 20-October-2019; Revised: 23-December-2019; Accepted: 26-December-2019

C2019 Mohammed Khaled Mustafa and Sajjad Waheed. This is an open access article distributed under the Creative Commons Attribution (CC BY) License, which permits unrestricted use, distribution, and reproduction in any medium, provided the original work is properly cited.

\begin{abstract}
Integrity and transparency are vital ingredients in both private and public sectors, especially in the matter of the tendering process. Government agencies and private organizations are continuously working to bring fairness in this process. The e-Tendering process brings efficiency and convenience in the overall practices which come out in the seen recently. With the emergence of blockchain technology, researchers identified beneficial features for ensuring transparency in the tendering process. Combining with smart contracts it paved the way for a secure, immutable and auditable e-Tendering processes yet maintaining strong veracity. In this paper, the drawbacks of the Ethereum-based blockchain e-Tendering process is identified and a framework with the permissioned blockchain was proposed to overcome the drawbacks. The overall tendering scheme is modulated in the framework such a way that confidentiality, integrity, and transparency are ensured in the process. The framework can mitigate most of the present technical and procedural limitations in Ethereum based e-Tendering. It has added new features and may enhance the security also.
\end{abstract}

\section{Keywords}

Blockchain, Tendering, Hyperledger fabric, Chaincode, Transparency, Security.

\section{Introduction}

The electronic tendering process is rapidly growing popularity among private and public sectors for its efficiency and convenience. An end to end fair and transparent tendering process is desirable for all stakeholders and the proper business environment. Researchers and business entities are continuously working to improve its quality. In general, while offering a tender, an enterprise usually maintains the following steps [1].

1. An enterprise or a tendering organization opens a tender, and once it is available for bidding it remains unchanged till the end of the process.

2. Each tender includes a list of evaluation criteria based on legal, technical and financial requirements for selecting the best possible bidder.

3. Only the authorized bidding organizations can submit a bid, the contents of their bid will remain confidential throughout the tendering process and intact.

*Author for correspondence
The tendering organization will ensure that uninvited third parties will not be able to participate on behalf of authorized bidding organizations.

4. The overall tendering process should maintain confidentiality, such a way that the participating bidders will have no idea about each other's candidacy

5. Once the deadline is over, the tendering organization can open the offers submitted by the bidders. Any person in the tendering enterprise will have no idea about the financial offer of any bidder until the deadline of submission.

6 . The best bidder will be selected based on the financial offer and its compliance with the required criteria. The losing bidder can compare their bid with the winner to be confident about the fairness of the evaluation process.

7. The overall process should be auditable to concern stakeholders for transparency.

The technology should provide such a framework for the tendering process that confidentiality, integrity, 
and availability will be achieved, along with the auditability of every activity. The overall process has to be fair and transparent and the stakeholders should have full confidence in it.
A blockchain-based smart contract framework is suitable for e-tendering to achieve desired criteria.

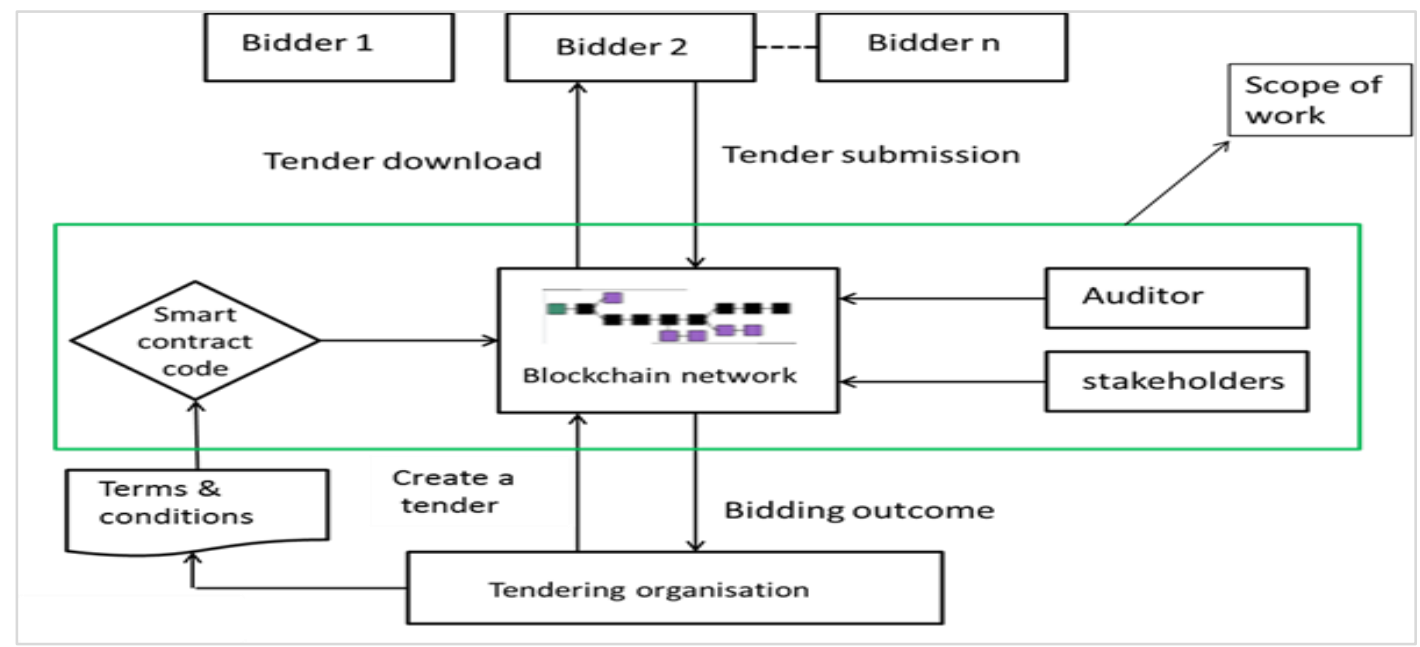

Figure 1 A standard blockchain-based smart contract system for e-tendering process

Figure 1 above is a high-level representation of blockchain-based on the e-Tendering process. It depicts the inclusion of the blockchain and smart contract technologies into the tendering framework. The diagram shows the different components of a blockchain-based e-Tendering process and shares how the flows are maintained among the traditional elements of the tendering system with the inclusion of smart contract. The scope of this work is also enclosed with a boundary mark in the figure.

The main objectives are as follows:

1. To review the existing blockchain-based system etendering systems and to find out the drawbacks of those systems.

2. To develop a new framework with permissioned blockchain to overcome the drawbacks.

3. To make propose a transparent e-tendering system by ensuring security, integrity and flexibility.

\subsection{Contribution}

In this paper, we proposed a unique e-Tendering framework based on permissioned blockchain. hyperledger fabric (HLF) is being used in the core of the system along with chaincode for business logic. The traditional tendering process is modulated with the system and transaction flow in the system is also shown here.

\subsection{Outline}

The rest of part of the paper is arranged as follows. In section 2, the background and review of earlier papers based on the blockchain-based e-Tendering process is discussed. The overall drawbacks and limitations of previous approaches are shown in the section. In section 3, a high-level overview is shared on HLF. The framework is presented in section 4 along with its different components and features. In section 5 transaction flow inside framework is displayed. The section 6 demonstrates the outcome and benefits of the framework. Lastly, the conclusion in section 7 summarizes the total contribution and identifies future research opportunity in this work.

\section{Background}

Blockchain technology is evolving rapidly and the type of solutions is also changing at a fast pace. There are few researches are already conducted on the tendering process with smart contract-based blockchain technology. The concept of the blockchain-based bidding system was discussed by Chen et al. [2]. It includes a smart contract in a simple e-bidding system to provide non-repudiation and confidentiality in the process. Ethereum based blockchain tendering framework for was shown by Hardwick [1]. Fairness and transparency in the tendering process along with the challenges of transaction cost or gas cost are focused there. The prospect of independent audit in the immutable blockchain ledger was also discussed in the paper. Though they demonstrated the proof of concept for blockchain-based e-tendering, the works lack in 
numbers of limitations and have some improvement scopes. The limitations are as follows:

1. Smart contracts must be written in a fixed and domain-specific language like Solidity for Ethereum based frameworks. It demands separate language expertise from the coders.

2. The frameworks are hardcoded and only useable for a single purpose. Once the purpose is over, they cannot be reused. Thus, it will be costly for enterprises.

3. The smart contract here cannot be updated or modified once the tendering process is launched. If any fault is identified or any change is inevitable then the whole tendering process has to be reinitiated.

4. In these Ethereum-based blockchain frameworks, all the transactions are done consecutively by all peers. It incurs a longer time for processing and mining costs to complete a transaction.

5. As smart contracts need to run on all the peers, it is difficult to maintain confidentially in case of domain-specific sensitive requirements.

In addition, the fairness in transaction with permissioned blockchain is discussed by Goel et al. [3]. The scheme showed a reference model implemented on HLF to capture the decentralization feature of blockchain and modularity of HLF.

The objective of this paper is to analyze the present challenges and shortcomings of Ethereum based etendering process and to develop a new framework with Permissioned blockchain that can ensure transparency and integrity. As the tendering process is closed one and confined only to a limited group of entities, we explored a suitable solution with private or enterprise blockchain backed by smart contracts for developing the desired framework for the organizations.

Blockchain platforms are mainly divided into two types:

- Public blockchain

- Private blockchain

The public blockchains are permissionless where anyone can join without prior permission and can become a member of the network. Bitcoin and Ethereum are examples of it. The Private blockchains, also known as enterprise blockchains, are always permissioned where only the authorized members are allowed to participate.
HLF and Exonum are examples of this type of blockchain. Through the design science approach [4] and reviewing the relevant journals and websites, it is evident that enterprise blockchains are suitable for our purposes as it has some inherent features to address the above-mentioned drawbacks of the Ethereum based system. Thereby, the HLF platform has been chosen for our framework. HLF has some key features which are suitable for our tendering frameworks. Those are as follows:

1. All participants must have prior authentication to join in the blockchain network.

2. It employs the execute-order-validate pattern which decouples the execution and endorsement of a transaction.

3. Mining process is not required here, hence anonymous miners are not required to validate transactions along with mining costs.

4. It can scale up with less latency as few peers can run the endorsement process instead of all the peers.

Before explaining the framework, some basic discussion on HLF will help for better understanding.

\section{Hyperledger fabric}

The HLF has a modular and flexible architecture. It supports open \& standard protocols. It can be found at [5-7] with different functionality.

HLF has the following basic modules as suggested in [5-7].

(a) Membership services: This module takes care of authentication and access control. It identifies which certification authorities (CAs) are trusted to define the members of a trusted domain and roles of peer in the network.

(b) Chaincode services: Smart contracts mainly contain the business logic of HLF. Chaincode is the superset of the smart contracts i.e. the smart contracts manage the business logic while Chaincode manages the smart contracts defined in it. All transactions run by Chaincodes are stored on the ledger.

(c) Consensus services: It permits digitally signed transactions to be proposed and validated by network members. In HLF consensus protocol is pluggable and interconnected to the endorse-order-validation process. The ordering services represent the consensus system in HLF. The guideline to authorize the validity of a transaction by any peer is defined in the endorsement policy. Besides the above module, there is some other key elements of HLF are as follows [8]: 
Ledger: Ledgers maintain chain of blocks that store all immutable historical records of every transition. Node: Nodes are the logical entities of the blockchain network. There are three types:

- Clients are used to submit transactions to the network through the users.

- Peers commit transactions in blockchain and preserve the ledger state.

- Orders create a communication channel between clients and peers;

Channels are isolated communication route for transferring confidential data between multiple network members. Activities on a channel are only visible to associated and authorized entities. Endorsers invoke smart contracts to validate transactions and send the results back to the applications.

\section{The framework}

The purpose of the framework is to control the eTendering process through enterprise blockchain features inside and permissioned environment and to achieve transparency and integrity throughout the process. The central component of the framework if HLF, which is described in the earlier section. The HLF maintains a business network connects the different business stakeholders through the client SDK (software development kit) in the tendering process. Figure 2 below shows the proposed framework along with their different components.

In a tendering organization, there are different parties like legal, sourcing, technology, etc. who set their specifications, terms and conditions for their corresponding perspective. Each of the business logic of the concerned department is coded through smart contract and chaincode manage and interconnect the smart contract as required. Here the framework ensures confidentiality of information between the functional units of both tendering and bidding organizations by maintaining separate channels of peers. For example, peers of technical domain can only check the compliance of technical specifications and requirements, but cannot access to financial or legal terms.

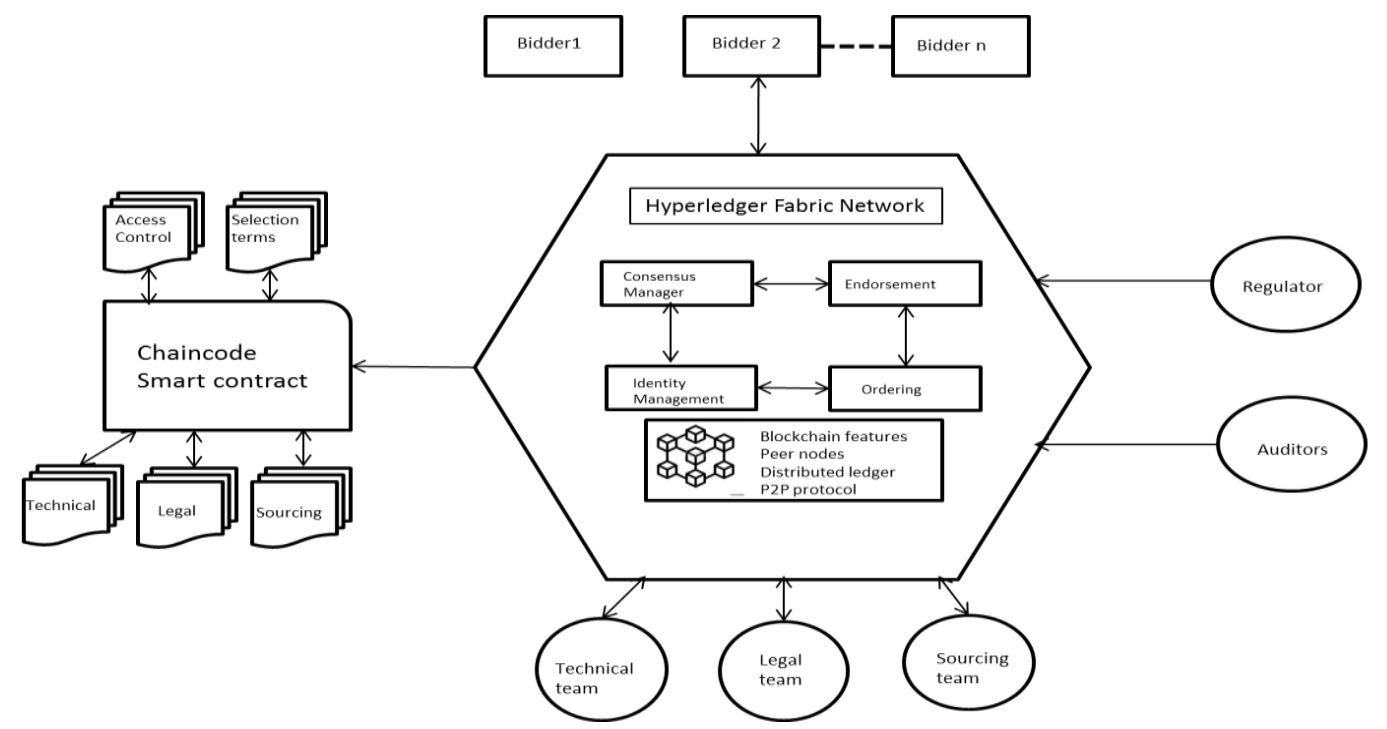

Figure 2 e-Tendering framework based on HLL

The bidding organizations collect and submit their documents through the client to the HLF SDK in the framework. The submitted documents are checked automatically by chaincode and results are available to the concern functions of tendering organization. As discussed earlier, there will be separate peers and channels for concern function so that an individual will only have the information which is related to him. In addition, regulators, auditor or any other concern party can access the framework based on their requirements to check the quality and compliance of the tendering process. Even anyone, internal or external to the organization, can monitor the process in real-time upon due consent. The ledger in the framework will remain immutable and no one can alter or damage the information event after a long time. The framework provides the flexibility and adaptability by utilizing the features of HLF so that it 
can be reused in the different tender process by introducing required chaincode and peers.

\section{Transaction flow}

The framework employs the execute-order-validate pattern which decouples the execution and endorsement of a transaction from its total ordering and committing to the ledger and creates the scope for parallel execution of independent transactions [9]. There are mainly three steps in the transaction life cycle in the framework $[5,6]$. Those are:

Execute: Transactions are executed through chaincode in any order, even in parallel in several channels. Order: After certain numbers of peers is agreed with the results of a transaction, it's then included in the ledger and distributed to all peers.

Validate: Each peer sequentially validates and applies transactions of the ledger. Now every transaction has an ordering so peers can validate the genuine transactions.

In the practical scenario, every bidder needs to connect with the framework through its client node. The client users and peer node members from both bidders and tendering organization have to be registered in membership service provider (MSP) and need digital certificates from the certification authorities (CA). At the beginning of the transaction, bidders will collect the tendering documents, review the requirements and will prepare their proposals. Now the transaction process flow according to the following steps in the framework.
Figure 3 shows the transaction flow inside the framework of a tendering process. The brief steps are discussed as per the following steps.

1. Bidder client initiates a transaction proposal along with its signature to the endorsing peer through the SDK.

2. The endorsing peers receive the transaction proposal from the bidder and verify it using the business logic written in chaincode and bidder's signature.

3. Once verified, the endorsing peers execute the transaction into the ledger and send a signed response back to the bidder's client.

4. The bidders receive and verify the response from endorsing peers. They have now submitted the responses to the orderer services through fabric SDK once again.

5. The orderer services sequentially order the transactions and arrange those in blocks. They deliver the blocks of the transaction channel-wise and send them to all the peers of specific channels.

6. The peers validate transactions in the blocks by reviewing signatures, version info and finally append the block to the blockchain. Afterward, the transaction is committed to the state database and notifies the application client.

In transaction flow, the steps $1,2 \& 3$ represent an endorsement process; the steps $4 \& 5$ resemble ordering process and the step 6 is like validation process.

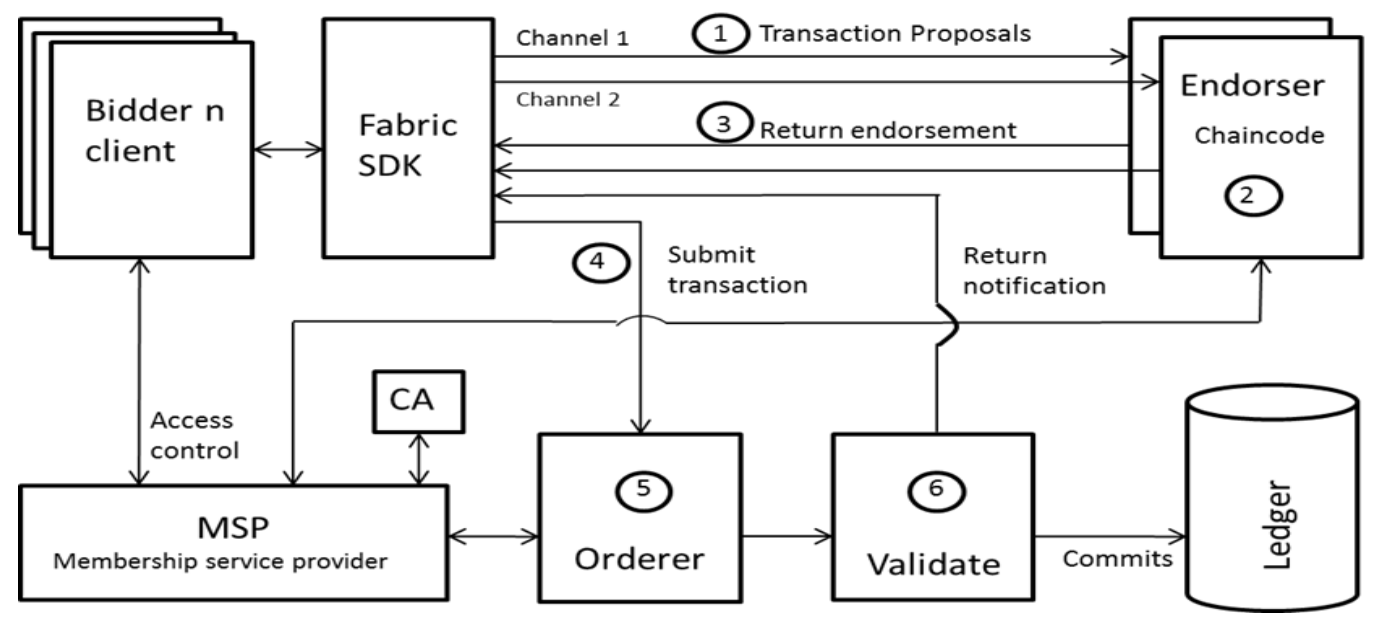

Figure 3 Transaction flow in the framework

After the timeline is over, only the authorized person of tendering organization can open the bidding documents and can select the best bidder for the 278 tendering organization through defined processes and algorithm controlled by chaincode and MSP. Besides, the losing bidders, the auditor and the regulator can 
review the overall process for clarification and quality checking.

\section{Discussion}

The framework addresses most of the drawbacks as mentioned in the background section. The points below sequentially share how the limitations of the Ethereum based system are mitigated in the HLF based framework.

1. In the framework, the smart contracts for chaincode can be written in traditional programming languages like Java, GO, etc. So, additional programming language skill is not required for the developer.

2. HLF is an open and standard enterprise blockchain operating system. The modular nature of its different components and chaincodes in Docker containers makes it versatile, flexible and reusable for many objectives.

3. All the chaincode in frameworks run on Dockers container $[9,10]$ so that it can be modified without interrupting the main process. Moreover, the introduction of discovery service in the HLF (v 1.2 or above) [4] makes it even more adaptable. So, any unavoidable changes can be accommodated in the framework with interrupting the main tendering process.

4. The framework can manage separate chaincode for separate types of peers through the channels. Here channels ensure isolation of business function by managing different blockchain. Moreover, as in the framework, only a few trusted nodes are enough to roll the transaction rather than waiting for every single node. So, the transaction is completed quickly and can be scaled up according to requirement. Besides, the traditional mining process is not required thus it saves both time and money too.

5. It ensures the adaptation of the practical process of the tendering system in the business network, with the separate involvement of legal, technical, sourcing team, etc. yet maintaining the privacy. MSP along with chaincode can control the access of individual users and can also set it a time-bound restriction. So, users cannot access beyond their stipulated access privilege and timeline. Moreover, all the activities are logged into append-only database in blocks and will remain immutable. Thus, it ensures the integrity, confidentiality, and non-repudiation of the overall process.

The framework will not only be limited to bring fairness and transparency in the tendering process but will also pave the way for other enterprise blockchain services like HRM, survey, inventory management and many more with the same infrastructure. In addition, it can also provide the necessary infrastructure for consortium blockchain [10] where multiple enterprises can work together for different projects using a common and shared platform.

Moreover, HLF is an off the shelf product which is maintained by the group of tech-giants like IBM, Linux Foundation, etc. So, the continuous development and expert support can be ensured for the enterprise will be available in this framework. As the permissioned blockchain allows limited access only to the trusted parties, the framework can ensure an extensive level of security and confidentiality of its process but no manipulation of information. It can also significantly reduce the risk of distributed denial-of-service (DDoS) attacks into the network with chaincode and MSP.

\subsection{Limitations and challenges}

Though the framework resolved numbers of drawbacks in the existing system apprehend some limitation and challenges in framework to implement. Those are as follows:

1. HLF is a very new technology and its features are rapidly changing. Building such a new network and making other stakeholders coping with it is the biggest challenge.

2. Scarcity of competent people on blockchain technology is another barrier to operate the system smoothly.

3. The practical implementation is not yet tested and checked.

\section{Conclusion}

In this paper, a conceptual framework for e-tendering with HLF has been developed. The framework was a unique approach to develop an e-Tendering process with an 'off the shelf' permissioned blockchain network like HLF. The benefits of the framework have been identified and discussed accordingly. The framework developed here has significant potential to bring healthy practices in the business networks. Lastly, the underneath objective of the paper was to device a technological solution so that undue exploitation of the tendering system can be reduced.

\section{Future directions}

However, this paper opens new scopes of future research in several areas. To be precise,

1. The mining cost and computation complexities are eliminated in the framework compared to the Ethereum based system. An analysis of operational 
benefits (e.g. gas cost, time, etc.) can be conducted to evaluate the actual gain.

2. The inclusion of the 'Blind Signature' feature with the framework could make the tendering process even fairer, unbiased and transparent.

3. Developing new capability like e-voting or privacy preserving survey could be future scope to explore with the framework.

\section{Acknowledgment}

None.

\section{Conflicts of interest}

The authors have no conflicts of interest to declare.

\section{References}

[1] Hardwick FS, Akram RN, Markantonakis K. Fair and transparent blockchain based tendering framework-a step towards open governance. In international conference on trust, security and privacy in computing and communications international conference on big data science and engineering (TrustCom/BigDataSE) 2018 (pp. 1342-7). IEEE.

[2] Chen YH, Chen SH, Lin IC. Blockchain based smart contract for bidding system. In international conference on applied system invention 2018 (pp. 208-11). IEEE.

[3] Goel S, Singh A, Garg R, Verma M, Jayachandran P. Resource fairness and prioritization of transactions in permissioned blockchain systems (Industry Track). In proceedings of the international middleware conference industry 2018 (pp. 46-53). ACM.

[4] Manevich Y, Barger A, Tock Y. Service discovery for hyperledger fabric. In proceedings of the ACM international conference on distributed and eventbased systems 2018 (pp. 226-9). ACM.

[5] Corten P. Implementation of blockchain powered smart contracts in governmental services. Delft University of Technology. 2018.

[6] Nawari NO, Ravindran S. Blockchain technology and BIM process: review and potential applications. Journal of Information Technology in Construction (ITcon). 2019; 24(12):209-38.
[7] https://www.hyperledger.org/projects/fabric. Accessed 10 August 2019.

[8] https://hyperledger-fabric.readthedocs.io. Accessed 10 August 2019.

[9] Androulaki E, Barger A, Bortnikov V, Cachin C, Christidis K, De Caro A, et al. Hyperledger fabric: a distributed operating system for permissioned blockchains. In proceedings of the thirteenth eurosys conference 2018 (p. 30). ACM.

[10] https://composer-playground.mybluemix.net/editor. Accessed 10 August 2019.

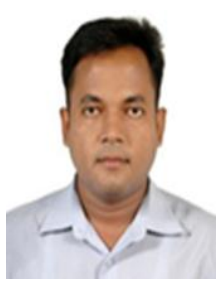

Mohammed Khaled Mustafa is an M.Sc. student of Master in Information Systems Security (MISS) program under the Department of Information and Communication Technology of Bangladesh University of Professionals. He has completed B.Sc. in Electrical and Electronics Engineering from Bangladesh University of Engineering and Technology. Currently, he is working with a Leading Telecommunication Operator in Bangladesh. His areas of interest are Blockchain Technology, E-Voting, ETendering and Information Security.

Email: khaled95@gmail.com.

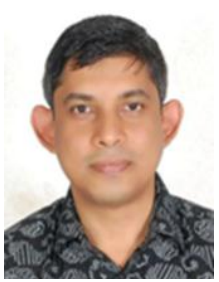

Dr. Sajjad Waheed currently works as a Professor at the Department of Information and Communication Technology, Mawlana Bhashani Science and Technology University, Tangail, Bangladesh. He is a Ph.D. holder in the field of Computer Engineering from Istanbul University Turkey. He has research interest in Machine Learning, Artificial Intelligence, Artificial Neural Network Data Mining and Blockchain Technology. 